Endocrinol. Japon. 1989, 36 (5), 775-779

\title{
NOTE
}

\section{TSH-Binding Inhibitor Immunoglobulin (TBII) in Thyroidal Venous Blood and Histological Grade of Intrathyroidal Lymphocyte Appearance in Graves' Disease}

\author{
Yoshio KASUGA, Akira SUGENOYA, Shinya KOBAYASHI, \\ Hiroyuki MASUDA, Minoru FUJIMORI AND Futoshi IIDA \\ Department of Surgery, Shinshu University School of Medicine, \\ Matsumoto 390, Japan
}

\begin{abstract}
The aim of this study is to investigate the possibility of determining the appropriate amount of thyroid tissue to leave behind as remnant weight in subtotal thyroidectomy for Graves' disease by measuring the TBII value, and correlating the outcome to the grade of intrathyroidal lymphocyte infiltration.

We therefore have investigated the levels of TBII in the thyroidal (T-TBII) and peripheral (P-TBII) venous blood, and the grade of lymphocytic infiltration and lymph follicle formation in the intrathyroidal tissue. There was no parallel relationship between the T-TBII value and the grade of 1ymphocytic infiltration and lymph follicle formation. There was no marked difference between the T-TBII value and the P-TBII value. It was thus not possible to use these data to determine the proper remnant weight of Graves' thyroid tissue at the time of surgery.
\end{abstract}

Current evidence supports the view that TSH-binding inhibitor immunoglobulin (TBII) observed in Graves' disease (GD) is produced mainly by the intrathyroidal B-lymphocytes in response to TSH receptorrelated antigens (Weetman et al., 1982; Kendall-Taylor et al., 1984; Volpé, 1985; McLachlan et al., 1986). If so, it would seem reasonable that the magnitude of synthesis of TBII might relate to the histological grade of lymphocytic infiltration and lymph follicle formation within the thyroid gland. If these relationships were true, the TBII value might prove useful in

Received June 27, 1989

Correspondence: AKIRA SUGENOYA M. D., Department of Surgery, Shinshu University School of Medicine, 3-1-1 Asahi, Matsumoto 390, Japan deciding the proper weight of thyroid tissue to be left behind (remnant weight) in the surgical treatment of persons with GD, since the benefits of thyroidectomy might depend, at least in part, on the removal of thyroidal lymphocytes which would be the prime source of TBII.

Therefore, the aim of this study was to investigate the relationship between the TBII value in the thyroidal vein (T-TBII) and the grade of lymphocytic infiltration and lymph follicle formation of the thyroid, and the relationship of such data to the weight of the gland at the time of surgery in patients with Graves' disease.

It is also of interest that some investigators have reported that the T-TBII value is higher than the TBII value in the peripheral veins (P-TBII) (Kendall-Taylor et al., 
1984; Carmen et al., 1986). However, a recent report has shown that there is no difference between the T-TBII values and P-TBII (Wilson et al., 1988). We have herein confirmed the observation of Wilson et al. (1988) and find no relationship between the TBII and the degree of intrathyroid lymphocyte infiltration.

\section{Materials and Methods}

\section{Subjects}

Thirty-one preoperative patients with GD were studied-four men and twenty-seven women with ages ranging from fifteen to eighty-seven years. All patients studied were diagnosed by

Table 1. Correlation between TBII values in thyroidal and peripheral venous blood and the histological grade of intrathyroidal lymphocytic infiltration (LI) and lymph follicle formation (LF)

\begin{tabular}{|c|c|c|c|c|c|c|}
\hline \multirow{2}{*}{ Case } & \multirow{2}{*}{$\begin{array}{c}\text { Age } \\
\text { (y.o.) }\end{array}$} & \multirow{2}{*}{ Sex } & \multirow{2}{*}{$\begin{array}{l}\text { Total } \\
\text { weight } \\
\text { (g) }\end{array}$} & \multicolumn{2}{|c|}{ TBII values $(\%)$} & \multirow{2}{*}{$\begin{array}{l}\text { Grade of } \\
\mathrm{LI} \text { and } \mathrm{LF}\end{array}$} \\
\hline & & & & Peripheral vein & Thyroidal vein & \\
\hline 1 & 23 & $\mathrm{~F}$ & 24 & 9.5 & 0.0 & (H) \\
\hline 2 & 33 & $\mathrm{~F}$ & 25 & 15.8 & 21.6 & (H) \\
\hline 3 & 32 & $\mathrm{~F}$ & 28 & 27.6 & 26.1 & $(+)$ \\
\hline 4 & 20 & $\mathrm{~F}$ & 28 & 52.9 & 32.6 & $(-)$ \\
\hline 5 & 17 & $\mathrm{~F}$ & 31 & 47.0 & 47.4 & (H) \\
\hline 6 & 29 & $\mathrm{~F}$ & 33 & 82.2 & 84.6 & (卅) \\
\hline 7 & 48 & $\mathrm{~F}$ & 34 & 17.2 & 17.2 & $(+)$ \\
\hline 8 & 31 & $\mathrm{~F}$ & 35 & 22.6 & 24.0 & $(-)$ \\
\hline 9 & 31 & $\mathrm{~F}$ & 38 & 5.6 & 5.5 & $(H)$ \\
\hline 10 & 32 & $\mathrm{~F}$ & 39 & 32.3 & 32.7 & $(+)$ \\
\hline 11 & 19 & $\mathrm{~F}$ & 46 & 52.5 & 55.3 & $(-)$ \\
\hline 12 & 33 & $\mathbf{M}$ & 47 & 31.4 & 28.8 & $(-)$ \\
\hline 13 & 39 & $\mathbf{M}$ & 48 & 29.7 & 33.6 & $(-)$ \\
\hline 14 & 49 & $F$ & 49 & 1.0 & 1.0 & $(-)$ \\
\hline 15 & 28 & $\mathrm{~F}$ & 53 & 75.6 & 77.8 & $(-)$ \\
\hline 16 & 38 & $F$ & 57 & 29.7 & 33.6 & $(-)$ \\
\hline 17 & 15 & $\mathbf{M}$ & 58 & 63.4 & 65.3 & $(H)$ \\
\hline 18 & 28 & $\mathrm{~F}$ & 60 & 14.1 & 13.3 & $(H)$ \\
\hline 19 & 40 & $\mathrm{~F}$ & 62 & 83.8 & 83.4 & $(H)$ \\
\hline 20 & 31 & $\mathrm{~F}$ & 67 & 39.8 & 63.8 & $(+)$ \\
\hline 21 & 56 & $\mathrm{~F}$ & 74 & 9.1 & 9.4 & $(+)$ \\
\hline 22 & 40 & $\mathrm{~F}$ & 75 & 20.2 & 22.9 & $(-)$ \\
\hline 23 & 25 & $\mathrm{~F}$ & 76 & 1.1 & 1.9 & $(H)$ \\
\hline 24 & 22 & $\mathbf{M}$ & 76 & 52.8 & 52.1 & (H) \\
\hline 25 & 31 & $\mathrm{~F}$ & 83 & 0.1 & 18.3 & $(+)$ \\
\hline 26 & 87 & $\mathrm{~F}$ & 90 & 72.3 & 69.8 & (H) \\
\hline 27 & 48 & $F$ & 94 & 80.4 & 79.9 & $(H)$ \\
\hline 28 & 19 & $\mathrm{~F}$ & 96 & 31.2 & 28.1 & $(+)$ \\
\hline 29 & 46 & $\mathrm{~F}$ & 118 & 14.1 & 14.9 & $(+)$ \\
\hline 30 & 48 & $\mathrm{~F}$ & 193 & 55.3 & 56.0 & $(-)$ \\
\hline 31 & 59 & $\mathrm{~F}$ & 282 & 38.6 & 37.2 & $(\mathrm{H})$ \\
\hline
\end{tabular}

* Grade of LI and LF

(H) High

(H) Moderate

$(+)$ Low

(-) Negative 
accepted clinical and laboratory criteria. For the operative preparation, the anti-thyroid drugs (methimazole and propylthiouracil) or corticosteroids combined with iodides were employed.

\section{Blood samples}

Blood samples from a peripheral vein and a thyroid vein were taken immediately after surgical exposure of the whole gland.

\section{Immunological tests}

TBII values were measured with a Smith's RIA kit (R.S.R. Ltd., Cardiff, United Kingdom) (Southgata et al., 1984). All TBII values above $15 \%$ were defined as positive according to the values provided in the kit.

\section{Histological observation}

As the correlation between lymphocytic infiltration and lymph follicle formation was almost paralle1, both of these features are arranged together in the results. The grade of lymph follicle formation in the thyroidal tissue was classified into four categories as follows : high : more than 5 1ymph follicle formations/field $(\times 40)$; moderate: one to four lymph follicle formations/field; low: one or two lymph follicle formations/several fields and negative: no lymph follicle formation found.

\section{Results}

The relationship between $\mathrm{T}$-TBII values and P-TBII values, and the histological grade of intrathyroidal lymphocytic infiltration and lymph follicle formation are shown in Table 1. Twenty-four out of 31 patients $(77 \%)$ had positive T-TBII values and seven were negative. There was no marked difference between $\mathrm{T}$-TBII values and P-TBII values in 21 out of 24 patients (88\%) with positive T-TBII.

Those findings on lymphocytic infiltration and lymph follicle formation will be mentioned in detail below. Table 2 shows the summary of the correlation between T-TBII values and the grade of lymphocytic infiltration and lymph follicle formation. There existed no parallel relationship between the TBII values and the grade of lymphocytic infiltration and lymph follicle formation.

The correlation among total thyroidal weight, T-TBII values and the grade of lymphocytic infiltration and lymph follicle formation is summarized in Table 3 . There was no parallel relationship among them.

Table 2. Relation between TBII values in thyroidal venous blood and grade of lymphocytic infiltration (LI) and lymph follicle formation (LF)

\begin{tabular}{|c|c|c|}
\hline TBII values & Grade of LI and LF & Cases \\
\hline \multirow[t]{5}{*}{ Positive } & & 24 \\
\hline & (卅) & 6 \\
\hline & $(H)$ & 3 \\
\hline & $(+)$ & 6 \\
\hline & $(-)$ & 9 \\
\hline \multirow[t]{5}{*}{ Negative } & & 7 \\
\hline & ( $\#)$ & 0 \\
\hline & $(H)$ & 4 \\
\hline & $(+)$ & 2 \\
\hline & $(-)$ & 1 \\
\hline
\end{tabular}

Table 3. Relation among total thyroidal weight, TBII values in thyroidal venous blood and grade of lymphocytic infiltration (LI) and lymph follicle formation (LF)

\begin{tabular}{|c|c|c|c|c|c|c|}
\hline \multirow{2}{*}{ Total weight (g) } & \multirow{2}{*}{ TBII values } & \multirow{2}{*}{$\begin{array}{l}\text { Total no } \\
\text { of cases }\end{array}$} & \multicolumn{4}{|c|}{ LI and LF } \\
\hline & & & (H) & $(+)$ & $(+)$ & $(-)$ \\
\hline \multirow{2}{*}{$<40$} & Positive & 8 & 3 & 0 & 3 & 2 \\
\hline & Negative & 2 & 0 & 2 & 0 & 0 \\
\hline \multirow{2}{*}{$40 \sim 80$} & Positive & 10 & 1 & 2 & 1 & 6 \\
\hline & Negative & 4 & 0 & 2 & 1 & 1 \\
\hline \multirow{2}{*}{$>80$} & Positive & 6 & 2 & 1 & 2 & 1 \\
\hline & Negative & 1 & 0 & 0 & 1 & 0 \\
\hline
\end{tabular}




\section{Discussion}

While TBII does not necessarily equate to thyroid stimulating antibody (TSAb), nevertheless the relationship is close in GD. TBII in this context may be considered to be responsible for the hyperthyroidism of GD. Most of the TBII is thought to be produced by TSH-receptor-sensitized lymphocytes within the thyroidal tissue (Weetman et al., 1982; Kendall-Taylor et al., 1984; Volpé, 1985; McLachlan et al., 1986), although there are reports that the extrathyroidal lymphoid sites produce autoantibody in GD (Weetman et al., 1984; McLachlan et al., 1986). It was thus speculated that the values for TBII might be relatively parallel to the grade of lymphocytic infiltration and lymph follicle formation in the thyroid tissue, although the intrathyroidal lymphocytes might have other functions aside from producing TBII. However, the relationship between TBII production and the intrathyroidal appearance of the lymphocytes had not been previously clarified, nor has a relationship between other thyroid autoantibodies and the degree of lymphocyte infiltration been elucidated in this fashion.

In this study, there was no apparent correlation between T-TBII values and the histological grade of lymphocytic infiltration and lymph follicle formation in thyroid tissue. Furthermore, there was no marked correlation between the total thyroidal weight and the grade of lymphocytic infiltration and lymph follicle formation or TBII production. Therefore, we assumed that both the specific antibody production and the functional quality of the lymphocytes were more important than the quantity of intrathyroidal lymphocytes, in terms of their immunological activity. It thus seems to be difficult to determine the proper remnant weight of thyroid tissue in the surgical treatment of person with
GD from the standpoint of the grade of lymphocytic infiltration and lymph follicle formation in thyroid tissue, and the TBII values.

On the other hand, there was no marked difference between the T-TBII values and $\mathrm{P}$-TBII values in 21 out of the 24 patients (88\%) showing positive T-TBII values, a result similar to that of Wilson et al., (1988). It seems likely that this finding might be due to the very prolonged (approximately 20 days) half-time of degradation of $\mathrm{IgG}$, which would then obscure any differences between thyroidal and peripheral venous TBII values. Another factor which might partially explain our results is that there may be some immunological suppression of TBII production by intrathyroidal lymphocytes due to preoperative antithyroid drug preparation at the time of surgery. It is also possible that there might have been a difference between T-TBII value and $P$-TBII value in the untreated condition.

We concluded that we were unable to determine the appropriate remnant weight of Graves' thyroid tissue by considering these data.

\section{Acknowledgments}

The authors wish to thank Professor Robert Volpé, University of Toronto, Toronto, Canada for critically reviewing the manuscript.

\section{References}

Carmen Arqueros, M., H. Niepomniszcze and J. Moreno (1986). Thyroid glands in patients with Graves' disease are sources of thyrotropinbinding inhibitor activity. Acta Endocrinol. 112, 351-355.

Kendall-Taylor, P., A. J. Knox, N. R. Steel and S. Atkinson (1984). Evidence that thyroidstimulating antibody is produced in the thyroid gland. Lancet i, 654-656.

McLachlan, S. M., C. A. S. Pegg, M. C. Atherton, 
S. L. Middleton, F. Clark and B. Ress Smith (1986). TSH receptor antibody synthesis by thyroid lymphocytes. Clin. Endocrinol. 24, 223-230.

Southgata, K., J. Creagh, M. Teece, C. Kingswood and B. Rees Smith (1984). A receptor assay for the measurement of TSH receptor antibodies in unextracted serum. Clin. Endocrinol. 20, 539-543.

Volpé, R. (1985). Autoimmune thyroid disease. In: Autoimmunity and Endocrine Disease (R. Volpé ed.), Marcel Dekker Inc., New York and Basel. pp. 109-285.
Weetman, A.P., A. M. McGregor, J. H. Lazarus and R. Hall (1982). Thyroid antibodies are produced by thyroid-derived lymphocytes. Clin. exp. Immunol. 48, 196-200.

Wilson, R., J. H. Mckilop, C. Pearson, A. K. Burnett, I. Gunn, A. M. McNicol and J. A. Thomson (1988). Absence of gradient of thyrotropin receptor antibody and $T$ cell subset distribution between thyroid and peripheral venous blood in patients with Graves' disease prepared for surgery with carbimazole and potassium iodide. Clin. exp. Immunol. 73, 265-268. 